\title{
Analysis of the time reserve influence on the technological cell productivity
}

\author{
Yuriy E. Obzherin ${ }^{1, *}$, Stanislav M. Sidorov ${ }^{1}$, and Sergey N. Fedorenko ${ }^{2}$ \\ ${ }^{1}$ Sevastopol State University, Higher mathematics Department, Sevastopol, Russia \\ ${ }^{2}$ Sevastopol State University, Department of Instrument Systems and Automation of Technological Processes, Sevastopol, Russia
}

\begin{abstract}
Time redundancy, widely used in technical systems, is one of the methods to improve the reliability and efficiency of technical systems. In this paper, the effect of instantly replenishing the time reserve on the productivity of a technical system is considered. Formulas of productivity of a technological cell in cases of random, constant and exponential time reserve are received, numerical results are given.
\end{abstract}

\section{Introduction}

Time redundancy $[1-10,16]$ is a method to improve the reliability and efficiency of technical systems, in which the system in the process of operation is given the opportunity to spend some additional time, called a reserve, to restore characteristics. The time reserve can be used for switching the structural reserve, detecting and eliminating system failures, repetition of work impaired by failures, waiting for the load to be operational. The presence of a time reserve is important in ergonomic systems [18] to correct possible operator errors.

A time reserve can be created by increasing the time allocated to the system for the task and called operational time. It occurs when you create a performance margin of the entire system or its individual devices, and without increasing the operational time. The presence of a time reserve is characteristic for technical systems that have a functional inertia $[3,9,11]$. The sources of the time reserve are intermediate stores, warehouses, reserve power sources.

One of the features of the time redundancy, which determines its dignity, is that improving the performance indicators of the system is often not related to the increase in the amount of equipment and the use of additional funds, but is based on the introduction or use of the time reserve available in the system itself.

For systems with time redundancy, a malfunction is not necessarily accompanied by a system failure, since there is the possibility to restore serviceability for a reserve time.

In most works, the influence of the time reserve on the reliability of the system is analyzed. In this paper, a semi-Markov model of a technological cell (TC) is constructed, taking into account the processing time of a unit of production and the presence of an instantly replenished time reserve. Using the constructed model, formulas for the productivity of the TC are found and the influence of the time reserve on productivity is analyzed.

\section{Description of the system}

We consider the functioning of the system S, consisting of a TC that processes products and has a random instantly replenished time reserve. At the initial time, the $\mathrm{TC}$ begins to process the products.

TC processing time for unit of production is a random variable (RV) $\alpha_{1}$ with the distribution function (DF) $\quad F_{1}(t)=P\left(\alpha_{1} \leq t\right)$ and distribution density (DD) $f_{1}(t)=\lambda_{1} e^{-\lambda_{1} t}$. TC time to failure is RV $\alpha_{2}$ with $\mathrm{DF}$ $F_{2}(t)=P\left(\alpha_{2} \leq t\right)$ and DD $f_{2}(t)=\lambda_{2} e^{-\lambda_{2} t}$. When the TC failure has occurred, the restoration of TC is beginning, TC restoration time is $\mathrm{RV} \beta$ with $\mathrm{DF}$ $\mathrm{G}(t)=P(\beta \leq t)$ and DD $\mathrm{g}(t)=\mu e^{-\mu t}$. Time reserve is $\mathrm{RV} \delta$ with $\mathrm{DF} \mathrm{R}(\mathrm{t})=\mathrm{P}(\delta \leq \mathrm{t})$ and $\mathrm{DDr}(\mathrm{t})$.

The failure of the system S occurs at the time when the recovery time of the TC becomes more than the time reserve and continues until the recovery of the TC. It is assumed that by the time of recovery of the TC, the time reserve is completely restored (instantly replenished time reserve).

In the event of a failure of the TC, the processing of the unit of production continues at the expense of the time reserve until the system fails. Then, after the recovery of the TC, the processing of a new unit of production begins. It is assumed that $\mathrm{RV} \alpha 1, \alpha 2, \beta, \delta$ are independent and have finite mathematical expectations.

To describe the system $\mathrm{S}$ operation, Markov renewal process (MRP) $\left\{\xi_{n}, \theta_{n} ; n \geq 0\right\}$ and corresponding semi-Markov process (SMP) $\xi(t)$ with discretecontinuous phase state space $[8,12,13,14,15]$ are used.

\footnotetext{
*Corresponding author: objsev@mail.ru
} 
Introduce the following set $\mathrm{E}$ of semi-Markov system states:

$$
E=\{1,10,11,20,21,1 \overline{0} x, 1 \overline{1} x, \omega\} .
$$

The meaning of state codes is the following: $1-\mathrm{TC}$ is able to work, the processing of unit production begins;

$10-\mathrm{TC}$ is able to work, the processing of a unit production is finished (instant state);

$11-\mathrm{TC}$ is able to work, the processing of a new unit of production begins;

20 - TC restoration has beginning, the processing of a unit production is continuing by the time reserve, system is able to work;

21 - TC restoration has finished, the processing of a unit production is continuing, time reserve is instantly replenished;

$1 \overline{0} x$ - TC restoration is continuing the processing of a unit production has finished by the time reserve, system is able to work (instant state); $\mathrm{x}$ is time till the end of TC restoration;

$1 \overline{1} x-\mathrm{TC}$ restoration is continuing the processing of a new unit of production begins by the time reserve, system is able to work; $x$ is time till the end of TC restoration;

$\omega$ - TC restoration is continuing, time reserve has finished, the processing of a unit production stopped, system failure.

\subsection{The system of integral equations for stationary distribution definition}

For finding the stationary characteristics of the system, it is important to find stationary distributions of embedded Markov chain (EMC) $\left\{\xi_{n} ; n>0\right\}$.

The general equation for the stationary distribution $\rho(B)$ is as follows:

$$
\rho(B)=\int_{E} \rho(d x) P(x, B),
$$

where $P(x, B)$-transition probabilities of EMC.

Denote $\rho(1), \rho(\omega), \rho(10), \rho(11), \rho(20), \rho(21)$ the values of EMC $\left\{\xi_{n} ; n>0\right\}$ stationary distributions in the states $1, \omega, 10,11,20,21$ and assume the existence of stationary densities $\rho(1 \overline{0} x), \rho(1 \overline{1} x)$ for the states $1 \overline{0} x, 1 \overline{1} x$ respectively.

Using formula (1) and the transition probabilities of the EMC, we obtain that the system of integral equations for the $\mathrm{EMC}\left\{\xi_{n} ; n>0\right\}$ stationary distributions looks like the following:

$$
\left\{\begin{array}{l}
\rho(1)=\rho(\omega)=\rho_{0}, \\
\rho(10)=\rho_{0} \frac{\lambda_{1}}{\lambda_{1}+\lambda_{2}}+\rho(11) \frac{\lambda_{1}}{\lambda_{1}+\lambda_{2}}+\rho(21) \frac{\lambda_{1}}{\lambda_{1}+\lambda_{2}}, \\
\rho(11)=\rho(10), \\
\rho(20)=\rho_{0} \frac{\lambda_{2}}{\lambda_{1}+\lambda_{2}}+\rho(11) \frac{\lambda_{2}}{\lambda_{1}+\lambda_{2}}+\rho(21) \frac{\lambda_{2}}{\lambda_{1}+\lambda_{2}}, \\
\rho(21)=\rho(20) \int_{0}^{\infty} \mu e^{-\mu y} e^{-\lambda_{1},} \bar{R}(\mathrm{y}) d y+\int_{0}^{\infty} \rho(1 \overline{0} y) d y \int_{0}^{y} \mu e^{-\left(\mu+\lambda_{1}\right) z} d z, \\
\rho(1 \overline{0} x)=\int_{x}^{\infty} \rho(1 \overline{0} y) \lambda_{1} e^{-\left(\mu+\lambda_{1}\right)(y-x)} d y+\rho(20) \int_{0}^{\infty} \lambda_{1} e^{-\left(\mu+\lambda_{1}\right) y} r(x+y) d y, \\
\rho(1 \overline{1} x)=\rho(1 \overline{0} x), \\
\rho(\omega)=\rho(20) \int_{0}^{\infty} e^{-\left(\mu+\lambda_{1}\right) \mathrm{y}} r(\mathrm{y}) d y+\int_{0}^{\infty} \rho(1 \overline{0} x) e^{-\left(\mu+\lambda_{1}\right) x} d x, \\
\rho(1)+\rho(\omega)+\rho(11)+\rho(10)+\rho(20)+ \\
+\rho(21)+\int_{0}^{\infty} \rho(1 \overline{0} x) d x+\int_{0}^{\infty} \rho(1 \overline{1} x) d x=1 .
\end{array}\right.
$$

The last equation of the system (2) is a normalization requirement.

\subsection{The case of a general time reserve}

Let the time reserve has general distribution $R(t)=P(\delta \leq t)$. By means of the method of successive approximations [12], the system of equations (2) is provided to have the following solutions:

$$
\left\{\begin{array}{l}
\rho(1)=\rho(\omega)=\rho_{0}, \\
\rho(10)=\rho_{0} \frac{\lambda_{1}}{\lambda_{2}} \frac{\lambda_{1}}{\lambda_{1}-\mu \int_{0}^{\infty} \bar{R}(t) h_{\tilde{g}}(\mathrm{t}) d t}, \\
\rho(11)=\rho(10), \\
\rho(20)=\rho_{0} \frac{\lambda_{1}}{\lambda_{1}-\mu \int_{0}^{\infty} \bar{R}(t) h_{\tilde{g}}(\mathrm{t}) d t}, \\
\rho(21)=\rho_{0} \frac{\mu \int_{0}^{\infty} \bar{R}(t) h_{\tilde{g}}(\mathrm{t}) d t}{\lambda_{1}-\mu \int_{0}^{\infty} \bar{R}(t) h_{\tilde{g}}(\mathrm{t}) d t}, \\
\rho(1 \overline{0} x)=\rho_{0} \frac{\lambda_{1}}{\lambda_{1}-\mu \int_{0}^{\infty} \bar{R}(t) h_{\tilde{g}}(\mathrm{t}) d t} \int_{0}^{\infty} \mathrm{r}(\mathrm{x}+t) h_{\tilde{g}}(\mathrm{t}) d t, \\
\rho(1 \overline{1} x)=\rho(1 \overline{0} x), \\
\lambda_{1}\left[\int_{0}^{\infty} e^{-\left(\mu+\lambda_{1}\right) t} r(t) d t+\int_{0}^{\infty} e^{-\left(\mu+\lambda_{1}\right) x} d x \int_{0}^{\infty} \mathrm{r}(\mathrm{x}+t) h_{\tilde{g}}(\mathrm{t}) d t\right]
\end{array},\right.
$$

where 
$\bar{R}(t)=1-R(t), \tilde{g}(t)=\lambda_{1} e^{-\left(\lambda_{1}+\mu\right) t}$,

$h_{\tilde{g}}(t)=\sum_{n=1}^{\infty} \tilde{g}^{*(n)}(t), \tilde{g}^{*(n)}(t)-n$th fold convolution of function $\tilde{g}(t)$. For function $h_{\tilde{g}}(t)$ has an equation

$$
h_{\tilde{\mathrm{g}}}(t)=\tilde{\mathrm{g}}(\mathrm{t})+\left(\tilde{\mathrm{g}} * h_{\tilde{\mathrm{g}}}\right)(t),
$$

which can be used to find it.

The value of constant $\rho_{0}$ is obtained from the normalization requirement and is not included in the final formulas of TC productivity.

For finding the productivity of the technological cell $\left(\mathrm{P}_{\mathrm{TC}}\right)$, we use the formula obtained in [17]:

$$
P_{T C}=\lim _{t \rightarrow \infty} \frac{N_{A}(t)}{t}=\frac{\int_{A} \rho(d x)}{\int_{E} m(x) \rho(d x)},
$$

where

$\rho(d x)$ is the EMC stationary distribution, which is defined by formula (3),

$\mathrm{m}(\mathrm{x})$ are average values of semi-Markov process sojourn times in states,

$\mathrm{N}_{\mathrm{A}}(\mathrm{t})$ - the number of visits of a semi-Markov process in a subset of states

$$
A=\{10 x, 1 \overline{0} x\} .
$$

When a semi-Markov process visits the states of this subset, the processing of a unit production ends.

The sojourn times in states of the system (2) are determined as follows:

$$
\begin{aligned}
& \theta(1)=\theta(21)=\theta(11)=\lambda_{1} \wedge \lambda_{2}, \\
& \theta(10)=\theta(1 \overline{0} x)=0, \\
& \theta(1 \overline{1} x)=\lambda_{1} \wedge \beta \wedge x, \theta(\omega)=\beta,
\end{aligned}
$$

where $\Lambda$ is the minimum sign.

Therefore, system average sojourn times $\mathrm{m}(\mathrm{x})$ in states have the form:

$$
\begin{aligned}
& m(1)=m(21)=\mathrm{m}(11)=\frac{1}{\lambda_{1}+\lambda_{2}}, \mathrm{~m}(10)=\mathrm{m}(1 \overline{0} x)=0, \\
& m(1 \overline{1} x)=\int_{0}^{x} \bar{F}_{1}(t) \bar{G}(t) d t, m(\omega)=M \beta .
\end{aligned}
$$

In this case, using the stationary distribution EMC (3) and system average sojourn times in states (6), the TC productivity is calculated by the formula:

$$
P_{T C}=\frac{\mu}{\mu+\lambda_{2}}\left(\lambda_{1}+\lambda_{2} \int_{0}^{\infty} \bar{R}(t) h_{\tilde{g}}(t) d t\right) .
$$

Using formula (7), we find the TC productivity formulas for particular cases of the time reserve distribution laws.

\subsection{The case of exponential time reserve}

Consider the case, when the time reserve is random and has an exponential distribution with DF $\mathrm{R}(t)=1-e^{-\tau t}$.

In this case the solution of system (2) has the form:

$$
\left\{\begin{array}{l}
\rho(1)=\rho(\omega)=\rho_{0}, \\
\rho(10)=\rho_{0} \frac{\lambda_{1}(\mu+\tau)}{\lambda_{2} \tau}, \\
\rho(11)=\rho(10) \\
\rho(20)=\rho_{0} \frac{\mu+\tau}{\tau}, \\
\rho(21)=\rho_{0} \frac{\mu}{\tau} \\
\rho(1 \overline{0})=\rho_{0} \frac{\lambda_{1}}{\tau} \\
\rho(1 \overline{1})=\rho(1 \overline{0})
\end{array}\right.
$$

Using the stationary distribution and formula (4), we obtain the formula for the productivity of the TC:

$$
P_{T C}=\frac{\lambda_{1} \mu\left(\lambda_{2}+\mu+\tau\right)}{\left(\lambda_{2}+\mu\right)(\mu+\tau)} .
$$

\subsection{The case of a costant time reserve}

Consider the case, where the time reserve is constant, namely $\delta=\tau=$ const. In this case $\mathrm{R}(t)=1(t-\tau)$.

The solution of system (2) can be written as follows:

$$
\left\{\begin{array}{l}
\rho_{0}=\rho(\omega)=\rho(1), \\
\rho(10)=\rho(11)=\rho_{0} \frac{\lambda_{1}}{\lambda_{2}} e^{\mu \tau}, \\
\rho(20)=\rho_{0} e^{\mu \tau}, \\
\rho(21)=\rho_{0}\left(e^{\mu \tau}-1\right), \\
\rho(1 \overline{0})=\rho(1 \overline{1})=\rho_{0} \frac{\lambda_{1}}{\mu}\left(e^{\mu \tau}-1\right) .
\end{array}\right.
$$

Substituting the obtained stationary distributions in (4), we have the following formula:

$$
P_{T C}=\frac{\lambda_{1}}{\mu+\lambda_{2}}\left(\mu+\lambda_{2}\left(1-\mathrm{e}^{-\mu \tau}\right)\right) \text {. }
$$

Let us analyze, for example, the effect of the time reserve on the TC productivity, using the formula (11).

Example. 


$$
\text { Let } \quad M \alpha_{1}=\frac{1}{12} \mathrm{~h}, \quad M \alpha_{2}=\frac{1}{2} \mathrm{~h}, \quad M \beta=\frac{1}{3} h,
$$
that is $\lambda_{1}=12(1 / h), \lambda_{2}=2(1 / h), \mu=3(1 / h)$, and the time reserve varies from 0 to 2 hours in 0.2 increments.

Figure 1 shows the influence of the time reserve on the productivity of the technological cell. In this case, the productivity of the TC without a time reserve is 7.2 units/hour. It follows from formulas (9) and (11), that the time reserve significantly affects on the productivity of the TC.

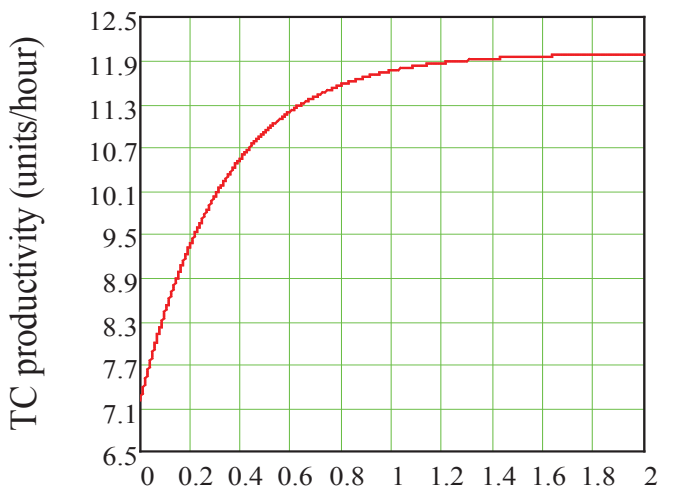

time reserve (hour)

Fig. 1. The influence of the time reserve on the productivity of the TC.

\section{Conclusions}

In this paper, a semi-Markov model of the technological cell, taking into account the processing time of a unit of production and the availability of an instantly replenished time reserve, is constructed. To construct the model, we used the theory of semi-Markov processes with a common phase space of states. A system of equations is constructed for the stationary distribution of the embedded Markov chain of a semi-Markov process and its solution is found by the method of successive approximations.

When the productivity of a technological cell is found, cases of exponential, general type and constant time reserve are considered. The analysis of the influence of the time reserve on the technological cell productivity in the case of a constant time reserve was carried out. It is shown that the presence of an instantly replenished time reserve significantly affects on the productivity of the technological cell.

We note the connection between the model considered and the random walk theory.

The results of this work can be used to analyze the influence of the time reserve on the productivity of technical systems for various purposes. The applied technique can be used in the construction of multicomponent systems with different types of time reserve.

To model multicomponent systems with a time reserve, it is proposed to use phase-coherence algorithms
[13], which are an effective tool for simplifying the analysis of complex systems.

The work has supported by a grant RFBF № 15-01-05840

\section{References}

1. G. N. Cherkesov, Reliability of Technical Systems with Time Redundancy (Sovietskoe Radio, Moscow, 1974) (in Russian)

2. B.P. Kredentser, Prediction of Reliability of Systems with Time Redundancy. (Naukova Dumka, Kiev, 1978) (in Russian)

3. Yu.N. Rudenko, I.A. Ushakov, Reliability of Energy Systems (Nauka, Novosibirsk, 1989) (in Russian)

4. Yu.E. Obzherin, A.I. Peschansky, Cybern. Syst. Anal., Vol. 30, No. 6. (1994)

5. Yu.E. Obzherin, A.I. Peschansky, Cybern. Syst. Anal., Vol. 40, No. 5. (2004)

6. Yu. E. Obzherin, A.V. Skatkov, J. Math. Sci., Vol. 57, No. 5. (2010)

7. I.A. Ushakov, Probabilistic Reliability Models (Wiley, 2012)

8. Y.E. Obzherin, E.G. Boyko, Semi-Markov Models: Control of Restorable Systems with Latent Failures (Elsevier Academic Press, London, 2015)

9. V.Y. Kopp, Yu. E. Obzherin, A.I. Peschanskiy, Stochastic models of automized system with time reservation (Publishing of Sevastopol State Technical University, Sevastopol, 2000) (in Russian)

10. G.N. Cherkesov, Reliability of hardware-software complexes, (Peter, St. Petersburg, 2005) (in Russian)

11. Lisnianski, A. and G. Levitin, H. Ben-Haim, Reliability Engineering \& System Safety, Vol. 67, Issue 2 (2010)

12. A.B. Vasileva, N.A. Tihonov, Integral equations ( Fizmatlit, Moscow, 2003) (in Russian)

13. V.S. Koroluk, A.F. Turbin, Markovian restoration processes in the problems of system reliability (Naukova Dumka, Kiev, 1982) (in Russian)

14. J. Jansen, N. Limnios (Eds.), Semi-Markov Models and Applications (Kluwer Academic Publishers, The Netherlands, 1999)

15. N. Limnios, G. Oprisan, Semi-Markov Processes and Reliability (Springer Science+Business Media, New York, 2001)

16. G.N. Cherkesov, Izv. AS USSR, Ser. Tehn. kibern., № 6, p.73-78 (1986)

17. V.M. Shurenkov, Ergodic Markovian processes (Nauka, Moscow, 1989) (in Russian)

18. E.A. Leontev, Reliability of economic information systems (Izd-vo Tamb. gos. tehn. un-ta, Tambov, 2002 ) (in Russian) 